\title{
Peer Buddy or Expert? - On the Avatar Design of a Virtual Coach for Obesity Patients
}

\author{
Thure Weimann \\ Technische Universität Dresden \\ thure.weimann@tu-dresden.de
}

\author{
Martin Fischer \\ Klinikum St. Georg gGmbH \\ martin.fischer@sanktgeorg.de
}

\author{
Hannes Schlieter \\ Technische Universität Dresden \\ hannes.schlieter@tu-dresden.de
}

\begin{abstract}
Morbid obesity in association with comorbidities is a considerable burden for the healthcare systems worldwide. Long-term weight loss maintenance requires sustainable behavioral changes but poor adherence is a significant problem in obesity care today and patients often relapse. Prior research has found conversational agents with of a humanoid representation (avatar) embodying the role of a virtual coach useful for the interface of health behavior change support systems. Regarding the avatar design, the coach could, e.g., take the role of an obese "peer buddy" or a lean "expert". Based on requirements and design principles derived from the literature, the present study investigates how the avatar should be designed. Therefore, two patient surveys were conducted to evaluate static and dynamic representations of potential coaches. The results suggest that patients welcome the concept and lean "expert" coaches might be more suitable in an obesity context. Design implications for future research are derived and discussed.
\end{abstract}

\section{Introduction}

According to a recent study, one in five deaths worldwide was associated with unhealthy nutrition [1]. Obesity, as a disease related to poor nutrition and high treatment costs, poses a significant challenge for the national health care systems [2]. The causative factors of obesity are manifold, with poor diet and a lack of physical activity playing an important role [3]. An increasing body mass index (BMI), increases the risk for secondary diseases as well (e.g., diabetes type 2, cardiovascular diseases or mental illnesses) and obesity becomes a complex chronic disease [3]. A BMI over 40 $\mathrm{kg} / \mathrm{m}^{2}$ is considered as morbid obesity and associated with a very high risk of comorbidities [4]. Sustainable lifestyle modifications with changes in nutrition and exercise behavior are mandatory for any long-term treatment success. Especially the life-long follow up care, characterized by a successive reduction of appointments carries a considerable risk of relapse. About $70 \%$ of patients relapse and do not maintain weight loss over a period of three years [5].

Health behavior change support systems (HBCSS), i.e., software applications that aim to transform attitudes or behavior by using persuasive techniques, promise an improvement of adherence and treatment outcomes [6][8]. Regarding the user interface of HBCSS, current research focuses on the development of conversational agents (CAs) taking the role of virtual coaches (VCs) [9]-[11]. CAs enable text or speech-based humancomputer interaction and are able to emulate interpersonal communication [12]. A subtype of CAs are embodied conversational agents (ECAs) that have an animated visual representation (also called avatar) [13], [14]. Prior research has revealed that in application areas in which rapport and trust are crucial (like health behavior change), ECAs should be preferred over disembodied agents [15]. Considering that demoralization due to a lack of social support and motivation are major barriers to long-term weight loss maintenance [16], ECAs as computerized social actors are a promising tool for obesity care. However, there is a lack of HBCSS that make use of avatar and CAtechnologies and address the needs of obese and especially morbidly obese patients. As a result, less research has been done on how the avatar should look like for this application context [17]. This is also surprising considering the stigmatization and selfstigmatization patients with obesity commonly experience [18]. A central question in this domain refers to whether the avatar should take the social role of an obese "peer buddy" or that of a lean role model. While a well-designed avatar can have a positive impact on the persuasion, imperfections may also lead to a rejection of the entire system [19]. Therefore, we want to investigate the following research question:

RQ: How should the avatar of a virtual coach for morbidly obese patients be designed? 
To address this research question, we followed a design science research (DSR) approach. The remainder of this paper is structured as follows. In the next section, we introduce the theoretical background of our study. Afterwards, we describe our research methodology in detail and present our results followed by a discussion. We close this paper with an outlook on future research and a summary of our contributions.

\section{Theoretical Background}

\subsection{Conversational Agents and Avatars for Health Behavior Change Support}

An essential foundation for using CAs and avatars as user interface of HBCSS is the "computers are social actors" paradigm [20]. The paradigm grounds the idea that humans apply the same rules to cues in software systems (e.g., trust as social response to a particular avatar design) as in human interactions [20]. Based on this seminal work and further research results, OinasKukkonen and Harjumaa [21] developed the persuasive systems design model that can be considered as one of the most important vehicles for guiding the development of HBCSS [7], [8]. The model proposes 28 design principles, where the present study examines the principle of employing the system with a "social role" more in detail and investigates how it can be applied to HBCSS in obesity care.

Prior research has found that different characteristics of the communicator such as likability, similarity, attractiveness, authority and credibility (with its key dimensions trust and expertise/intelligence) determine the persuasiveness and thus the intervention effectiveness [22], [23]. A question that has been discussed in the literature for years concerns whether a VC (or pedagogical agent in learning settings) similar to the user in terms of visual appearance ("peer") or an authoritative "expert" might be more effective [24] [27]. Several theories, such as the social cognitive theory [28] or similarity attraction theory [29] suggest that people might be more persuaded by similar others. Similar others can act as social models that demonstrate a certain target behavior and instill the awareness that the observer is able to successfully accomplish it too [30]. Furthermore, socially attractive communicators with a high degree of likability and similarity to the recipient are especially effective persuaders when people make personal and emotional decisions [22]. However, perceiving the communicator as an expert could be beneficial when facts are involved [27]. Consequently, the use of multiple coaches where each embodies a different role might be an effective approach and is referred to as "split persona" effect [31], [32].
With respect to the design of a $\mathrm{VC}$ for obesity patients, designing the avatar (or in case of multiple coaches at least one avatar) similar to the appearance of the user seems reasonable based on the previous considerations. Against the background that avatar technologies enable a realistic modeling of the individual's appearance, the maximum degree of similarity can be achieved if the avatar resembles a virtual “doppelganger". For instance, the study by Fox and Bailenson [33] investigated the influence of an avatar with the same face on physical activity behavior. The results revealed that observing how a virtual self model is running on a treadmill was more effective for increasing physical activity (in the real world) than observing a virtual other. However, the recent study by Navarro et al. [34] discloses that using avatars with real body dimensions can elicit anxiety in overweight people that are often unsatisfied with their own body image (mean BMI of study group $28.7 \mathrm{~kg} / \mathrm{m}^{2}$ ). In contrast, the study by van Vugt et al. [35] recommends the use of overweight avatars as virtual health advisors in general (mean of study group BMI $22 \mathrm{~kg} / \mathrm{m}^{2}$ ). Napolitano et al. [36] used obese avatars in educational videos in which the avatar demonstrates healthy eating and exercise behavior. The patients were then asked questions regarding their preference of an avatar with the same face and an avatar that changes the size/shape (becomes thinner) as the patient progresses through the program. The survey results $\left(n=8\right.$, mean BMI $\left.33 \mathrm{~kg} / \mathrm{m}^{2}\right)$ revealed that the participants had mixed preferences regarding an avatar that looks like them, while the majority preferred the idea of an avatar that simultaneously changes the body shape with the patient.

Since the study populations are significantly different from the target population of our study (BMI > $40 \mathrm{~kg} / \mathrm{m}^{2}$ ) it seems questionable to what extent the results of previous studies are applicable.

\subsection{Theory of Uncanny Valley}

While an anthropomorphic (human-like) software design can elicit positive social responses such as intelligence or trustworthiness [37], [38], mismatches of different anthropomorphic design elements (also called social cues) may lead to a rejection of the system. If the technology cannot meet the expectations that are aroused by a human-like design, the so called "uncanny valley" effect described in the theory by Mori et al. [39] may occur. The theory posits that increasing the "human-likeness" of an artifact can increase the acceptance at first, but the user feels eeriness by a system that appears "too human" but do not behave like a real human. Thus, the acceptance effect abruptly drops negative towards an "uncanny valley". Bartneck et al. developed the „Godspeed Questionnaire“, that is a scale 
widely used in robotic research to measure the user's perception of a robot. The questionnaire is available in several languages and assesses the dimensions anthropomorphism, animacy, likability, perceived intelligence and perceived safety of robots. However, due to correlations among these indices, the scale is only partially suitable for measuring the uncanny valley effect [40]. Therefore, a revised scale that comprises the dimensions eeriness, humanness and attractiveness has been suggested [40]. Both instruments are used in our research for evaluating design decisions.

\section{Research Methodology}

Our research follows the DSR framework proposed by Hevner [41] consisting of three design science cycles: relevance, design and rigor. The instantiated framework with the conducted steps is depicted in Figure 1. Based on this approach, we aim to systematically contribute to the knowledge base with new design implications [42]. The conducted research steps are embedded into a larger DSR project investigating the design of an avatar-based $\mathrm{VC}$ for obesity patients [43].

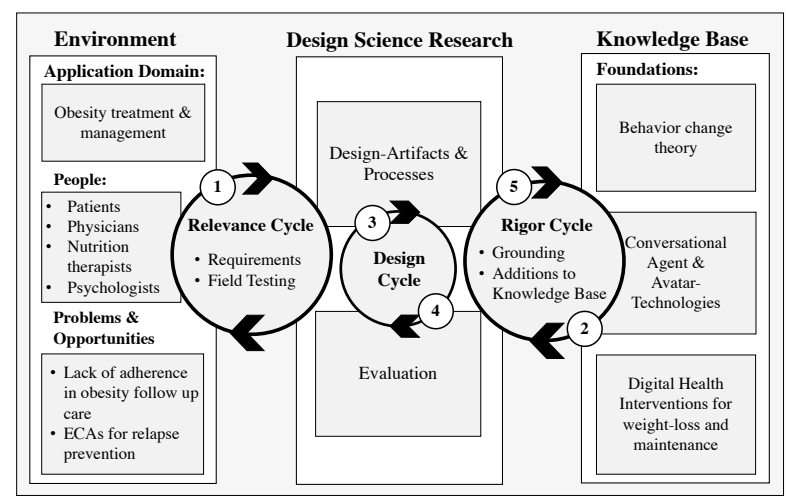

Research Steps:

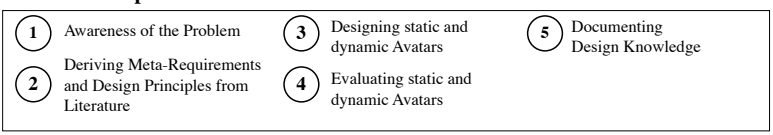

\section{Figure 1. DSR three cycle view according to Hevner [41] and conducted research steps}

We initialized our research with the awareness of the problem as described in the introduction (step 1). In the second step, we derived a first set of metarequirements and design principles from the literature (knowledge base). Against the background that several studies suggest that both static and dynamic aspects of the ECA influence the user's impression [25], [44], [45], we evaluated both perspectives. Therefore, we designed avatars of potential coaches based on the elicited requirements and design principles (step 3) and conducted an evaluation of the static and dynamic representation (step 4). After the evaluation, we documented our gathered design knowledge (step 5). The survey participants were recruited from a nonsurgical obesity treatment program in a German hospital. The program participants have a mean age of 46 years and a mean BMI of $49.5 \mathrm{~kg} / \mathrm{m}^{2}$ [16]. As our survey solely focused on opinions, a formal medical ethical approval was not required. All patients provided their consent.

The avatars of the potential coaches were rated on a semantic differential scale from 1 to 7 with regard to the dimensions: perceived humanness, eeriness, attractiveness, likability, perceived intelligence, trustworthiness, authority and the self-assessed likeliness following the coach's advice. The first three dimensions were adapted from the revised "Godspeed" scale proposed by Ho and MacDorman to assess the uncanny valley effect [40]. In order to avoid fatigue when completing the questionnaire, only the items with the highest factor load were considered. The likability and perceived intelligence scales were adapted with a subset of items from the Godspeed questionnaire by Bartneck et al. [46]. The remaining three dimensions (trustworthiness, authority and likeliness to follow the coach's advice) were adopted in accordance to the studies by ter Stal et al. [25], [47].

In addition, the patients were asked if they would prefer two coaches (peer and expert), what they think about losing weight simultaneously with the avatar (i.e., the avatar dynamically changes the BMI over time) and their preference regarding an avatar as virtual self (same hair, face,...). While the first question aimed to assess the general interest in a multi-coach approach (e.g., [48]), the second and third question were adopted in accordance to the study by Napolitano et al. [36]. Furthermore, a free-text field was included where the patients could express their own ideas.

\section{Deriving Meta Requirements and Design Principles from Scientific Literature}

As discussed in section 2, a persuasive design of the $\mathrm{VC}$ is of pivotal importance to digitally support behavioral changes [8], [11]. However, given that the avatar is always present to the user when interacting with the system, this feature poses a hurdle. In addition to the visual appearance, also design features such as speech or movements must be brought into harmony in order to ensure a persuasive appearance [38]. We summarize in MR-1: ,, The avatar of the virtual coach should be perceived persuasive". With respect to a persuasive design of the avatar, a rich body of design principles is available in the literature (e.g., [38], [49], [50]). Nevertheless, what the coach's avatar should look 
like in an obesity context is largely unclear. Opinions in literature differ and so far, to the best of our knowledge, no study has explored the appearance of an avatar-based $\mathrm{VC}$ in a morbidly obese population. Additionally, it is unclear to what extent a "split persona" (multi-coach) approach may be considered for a VC to support obesity patients. Such an approach has so far been less investigated, but can be found, e.g., in the recent Horizon 2020 project "Council of Coaches" (see [48]). For this reason, no meta-requirements and design principles can be stated here yet and should be examined more in detail in this work. While the similarity to the user is controlled via the BMI of the avatar in this study, previous studies revealed that perceived intelligence of the "expert" avatar can be triggered with social cues such as wearing eye-glasses [37] or a white doctor coat [51]. From these findings, we derived DP-1 using the structure proposed by Gregor et al. [52] (see Figure 2). To summarize, designers and researchers should equip the avatar with stereotypical cues which are also associated with experts in real life.

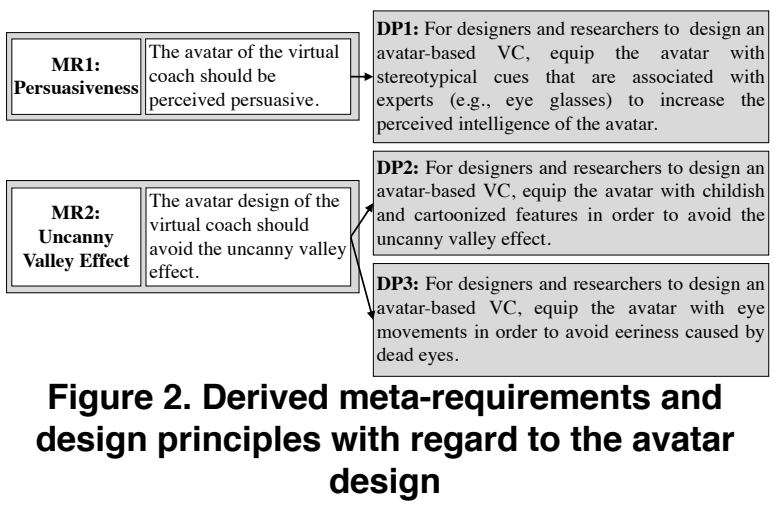

However, if various social cues appear "too human" or do not match with each other, feelings of uncanniness might be evoked (see section 2.2). Therefore, we state in MR-2 "The avatar design of the virtual coach should avoid the uncanny valley effect". For avoiding the uncanny valley effect, Schwind et al. [53] proposed several design principles. For example, several studies revealed that abstract representations and childish features, such as often found in cartoonized avatars (e.g. Disney Pixar) [54], [55] are useful for avoiding the uncanny valley. Another approach described by Schwind et al. [53] is to let the users themselves design the avatar (as it is often done in the gaming area) for increasing identification with the avatar. However, there is a lack of consensus regarding self-designed avatars in the ECA literature. Some authors argue that users tend to choose such that are not that suitable for them and thus, e.g., have negative impact on learning outcomes

\footnotetext{
${ }^{1}$ https://www.reallusion.com/character-creator/
}

[56], [57]. Other studies report that this approach could even have positive impact on the persuasion [58]. For this research, we address MR-2 by designing a cartoonized avatar and instantiated DP-2 based on [53][55]. In short, designers should equip the avatar with childish and cartoonized features for tackling the uncanny valley. Another design principle adopted from Schwind et al. [53] that is particularly relevant for dynamic avatars is the avoidance of dead eyes (see DP3 in Figure 2). Therefore, avatars equipped with eyemovements are less likely to be perceived as uncanny compared to static (dead) eyes.

\section{Designing static and dynamic Avatars}

Based on the derived requirements and design principles from literature, four static avatars of potential VCs were designed first. The avatars varied in terms of gender (male, female) and role (expert, peer). For representing the "peer buddy", the anthropometry was matched to the BMI of the target group, while the "experts" were designed with wearing eye glasses to stimulate perceived intelligence. Based on the study results by ter Stal et al. [25] that revealed that young avatars tend to be more preferred in a general population, we only designed young representations. However, it should be noted that there is no consent in the literature with regard to a preferable age of the avatar [38]. For designing the avatars, we used the software "Reallusion Character Creator 3" (CC3) that was extended by additional packages to design cartoonized avatars (see DP2) and enable a "Body Morphing" for increasing the BMI.

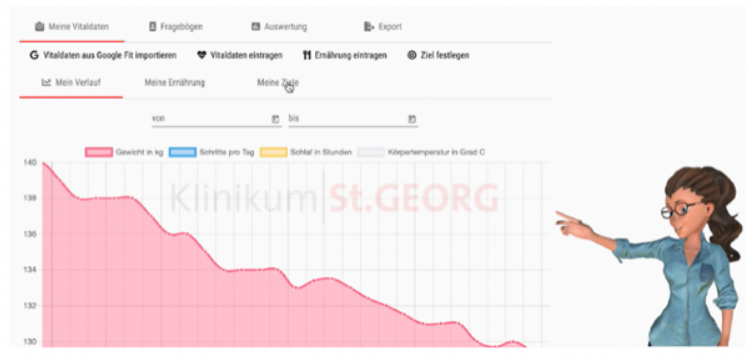

Figure 3. Feedback on the weight curve from the avatar-based coach

For implementing a dynamic version of the avatar, we used the package "SALSA LipSync Suite"2 that enables real-time lip sync approximation from liveinput and automates animation of eyes, eyelids and head. Likewise, the automated animations address the symptom of dead eyes (see DP-3). First, we exported the

\footnotetext{
${ }^{2}$ https://crazyminnowstudio.com/unity-3d/lip-sync-salsa/
} 
static avatar model with its textures (e.g., clothing, hair) designed in CC3 and imported the model into the gaming engine "Unity"3. In a next step, the mentioned package was added and linked to the avatar model. Additionally, several animations were added from the library "mixamo.com" as for example, greeting and pointing gestures or idle animations. Facial expressions were limited to a slightly smiling representation. Finally, we exported the avatar as $\mathrm{WebGL}^{4}$ build and integrated the avatar into a web application (see Figure 3).

\section{Evaluating static and dynamic Avatars}

First, the static avatars were evaluated in a patient group of $n=33$ (female: 23, male: 9, NA: 1) using a paper-based questionnaire. For validating the acceptance of the questionnaire, $n=5$ patients were surveyed first. After there were no problems indicated, the remaining patients were surveyed. The patients were asked two control questions regarding their general interest in using an avatar-based VC and their usage behavior of different technologies (computer, tablet, smartphone, smartwatch/fitness tracker) in their daily life. Of the $n=33$ participants, a total of $n=30$ could imagine to use an avatar-based VC. With respect to technology usage behavior, $n=32$ patients are using a smartphone, $n=18$ a computer, $n=10$ a tablet and $n=$ 23 a smartwatch/fitness tracker at least on a daily basis.
The results of the first survey are depicted in Figure 4 and 5 . In Figure 4 and 6, the mean value is white dotted highlighted and the median in a gray box. Differences were tested with a Wilcoxon test for paired samples (Bonferroni correction) and noticeable differences were marked. We used the Python package "scipy.stats" [59] for the tests.

The evaluation of static avatars (see Figure 4) shows that the obese peers were rated lower with respect to attractiveness, perceived intelligence, trustworthiness and likeliness to follow advice of the coach. The means of perceived humanness and eeriness were in the middle of the scale and differences between "peer buddy" and obese avatars did not reach statistical significance. With regard to the perceived humanness, the results correspond to the expectations come with a deliberately unrealistic but anthropomorphic designed avatar. Overall, the results suggest that an "expert" representation of the $\mathrm{VC}$ might be more suitable for morbidly obese patients. Asking the patients what they think about losing weight simultaneously with the avatar, reflects an ambivalent result. As depicted in Figure 5, a total of $n=25$ patients liked the idea of a dynamically changing avatar according to their body weight. With regard to the interest in a multi-coach approach and a virtual doppelganger, the results were mixed. However, one patient wrote in the free-text field: "The avatar should be similar to me and appear as a friend and help me to overcome my weaker self. [...] The avatar should lose weight with me and I would like to be

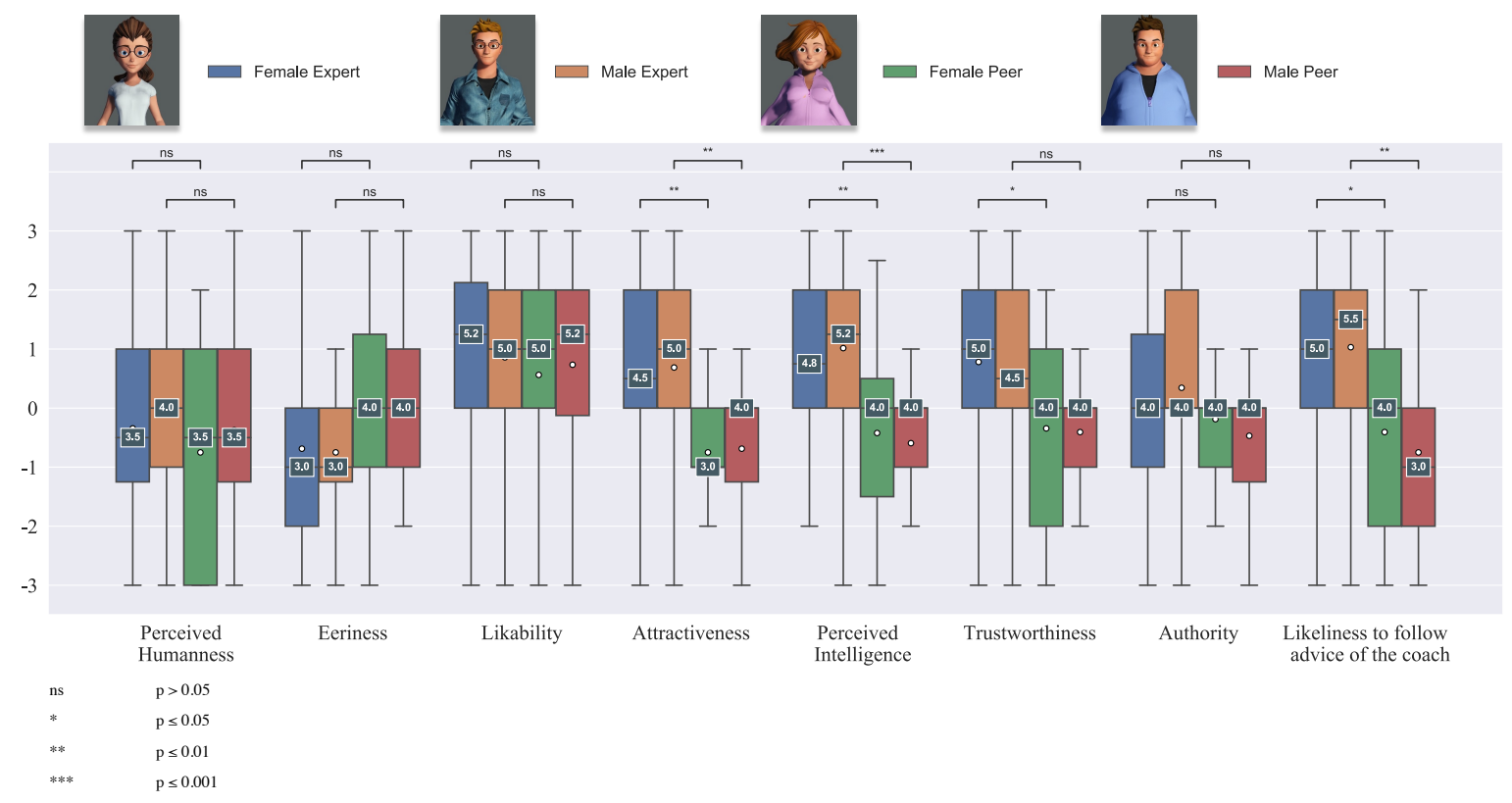

Figure 4. Ratings of potential coaches (static avatars)

\footnotetext{
${ }^{3}$ https://unity.com/
}

${ }^{4}$ https://www.khronos.org/webgl/ 
able to use the avatar to simulate how I look with less weight."

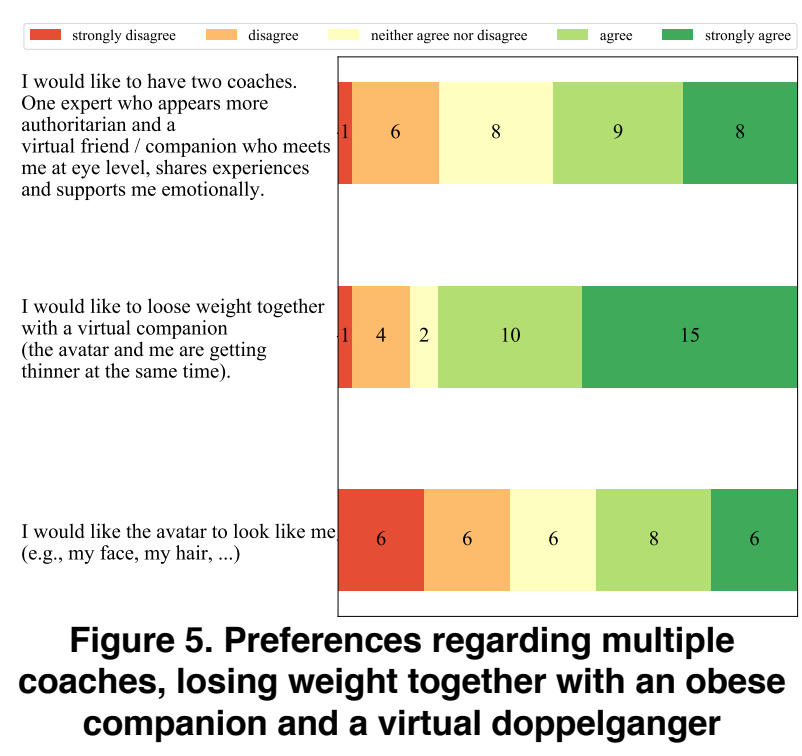

For the further study, we decided to initially evaluate only one dynamic avatar. Although the results indicate that male and female "expert" representations might be both suitable, a decision was made in favor of a female representation for the dynamic implementation and evaluation. The design decision is based on findings of previous studies revealing that female representations might be more beneficial in supportive relationship contexts (e.g., [25], [60]). In addition, there is a general bias with regard to the use of female representations in the research field of CAs [15], [61].

For evaluating the dynamic avatar, we chose a video-prototyping approach. Therefore, we created a one minute video clip $^{5}$ in which the $\mathrm{VC}$ presents the core functions of the system. First, the VC introduces herself ("Hello, my name is Lea. I'm your virtual coach...") and explains the weight and step curve, the nutrition diary and the goal-setting component (see Figure 3). We used a synthetic voice similar to the voice of the popular speech assistant "Alexa" from the text-tospeech service „Amazon Polly”.

A total of $n=12$ patients (female: 7, male: 5) participated in the dynamic evaluation. After the therapist presented the video, the patient filled out the same questionnaire as in the evaluation of static avatars. Figure 6 depicts the results. A two-sided Wilcoxon rank sum test against the neutral value of the scale showed significant differences $($ all $\mathrm{p}<.01)$. The results reveal that despite adding a synthetic voice to the avatar, the patients perceived the avatar as more human and not feel

\footnotetext{
${ }^{5}$ Video used for evaluation: https://youtu.be/EwJYHuewe9o
}

more eeriness compared to the static evaluation (see Figure 4). Additionally, the other dimensions also suggest that the avatar is perceived persuasive by the patients and thus, might be effective to support behavioral changes.

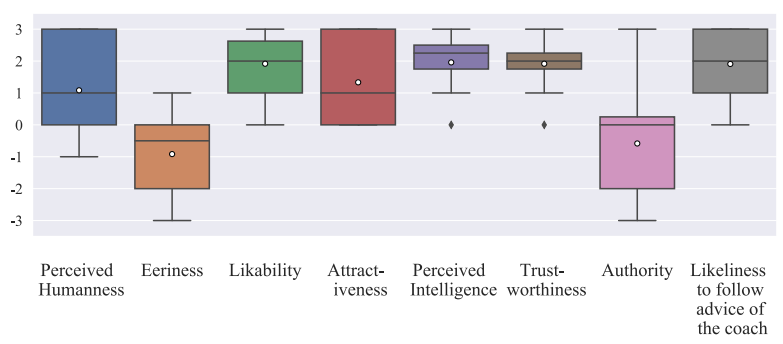

\section{Figure 6. Ratings of the dynamic avatar appearance}

\section{Discussing and Documenting the Design Knowledge}

Our DSR study aimed to investigate the design of an avatar as a VC to support morbidly obese patients in their daily life and foster behavioral changes. Against the background of disagreement in the literature, a research question of particular interest was whether the avatar should mirror the patients' obese appearance. Our results of the first design cycle suggest that the lean "expert" avatars were more suitable as VCs and preferred by the patients as compared to the obese "peer buddys". Even though the "peer buddy" approach is supported by several theories, such as the social cognitive [28] or similarity attraction theory [29], our results suggest that this might not apply to this patient group. However, our results confirm that deliberately cartoonized avatars are accepted by the patients and are potentially suitable as $\mathrm{VC}$. We found that patients were receptive towards the idea of being coached by multiple VCs. Implementing two VCs could be useful for patients to separate themselves from their destructive self, e.g., by creating one $\mathrm{VC}$ as the devil's advocate who challenges aspired cognitive-behavioral changes.

While it was expected that using stereotypical cues associated with experts (e.g., wearing eyeglasses) increases the perceived intelligence, the ratings with respect to attractiveness, trustworthiness and likeliness to follow advice of the coach showed significant differences too. One possible explanation for the rating differences could be that obesity patients are often dissatisfied with their own body and stigmatized in society [18], [62], [63]. However, the patients were

\footnotetext{
${ }^{6}$ https://aws.amazon.com/de/polly/
} 
interested in losing weight together with the avatar what would imply an obese appearance. This might be explained by the fact that the joint weight loss with the avatar visualizes one's own success. This is perceived as interesting from the patient's point of view but might be also achieved in form of a diagram visualizing the weight loss. Furthermore, our results are in line with the results of Napolitano et al. [36], where the participants were also interested in such a dynamic and also had mixed preferences regarding an avatar as virtual doppelganger. Based on the results, we propose DP4 that addresses MR1: "For designers and researchers to design an avatar-based VC for obesity patients, employ an obese avatar with consciousness, carefully evaluate the effects and be aware of body dissatisfactions and stigmatization in/of this patient group".

\section{Table 1. Documentation of the design knowledge based on Gregor and Jones [42]}

\begin{tabular}{|c|c|}
\hline Component & Description \\
\hline $\begin{array}{l}\text { Purpose and } \\
\text { scope }\end{array}$ & $\begin{array}{l}\text { The objective of an avatar-based VC for } \\
\text { morbidly obese patients is to provide support in } \\
\text { their daily life and foster sustainable behavioral } \\
\text { changes (esp. eating and exercise behavior) } \\
\text { with a persuasive system design. }\end{array}$ \\
\hline Constructs & $\begin{array}{l}\text { Eight constructs relevant for the avatar design } \\
\text { of the VC were derived from the literature: } \\
\text { perceived humanness, eeriness, attractiveness, } \\
\text { likability, perceived intelligence, trust- } \\
\text { worthiness, authority and likeliness to follow } \\
\text { advice of the coach }\end{array}$ \\
\hline $\begin{array}{l}\text { Principles of } \\
\text { form and } \\
\text { function }\end{array}$ & see DP1-DP4 \\
\hline $\begin{array}{l}\text { Artifact } \\
\text { mutability }\end{array}$ & $\begin{array}{l}\text { The avatar can be deployed on different } \\
\text { platforms (e.g., virtual or augmented reality } \\
\text { glasses, mobile phones or in the web-browser) }\end{array}$ \\
\hline $\begin{array}{l}\text { Testable } \\
\text { propositions }\end{array}$ & $\begin{array}{l}\text { H1: Lean "expert" avatars as VCs are perceived } \\
\text { as more intelligent, trustworthy, attractive and } \\
\text { patients tend to more likely follow their advice } \\
\text { compared to obese "peer buddy" avatars. }\end{array}$ \\
\hline & $\begin{array}{l}\text { H2: If the avatar design follows the derived } \\
\text { design principles, then a fall into the "uncanny } \\
\text { valley" is avoided. }\end{array}$ \\
\hline $\begin{array}{l}\text { Justificatory } \\
\text { knowledge }\end{array}$ & $\begin{array}{l}\text { Computers Are Social Actors Paradigm/Social } \\
\text { Response Theory [20], Theory of Uncanny } \\
\text { Valley [39], literature on the usage of VCs and } \\
\text { avatar technologies (esp. in weight-loss or } \\
\text { maintenance settings), literature on } \\
\text { investigations of social cues in the field of CAs }\end{array}$ \\
\hline
\end{tabular}

We summarized our gathered design knowledge in Table 1 based on the components of a design theory (purpose and scope, constructs, principles of form and function, artifact mutability, testable propositions and justificatory knowledge) as suggested by Gregor and Jones [42]. Thereby, we aim to systematically contribute to the knowledge base by providing a first guidance for designers and researchers in subsequent work. The present study particularly contributes with a set of metarequirements and design principles derived from the literature that were validated and extended with empirical investigations.

\section{Limitations and Opportunities for Future Research}

Although our study contributes with a first guidance on the avatar design of a HBCSS for morbidly obese patients, there are some limitations. First, we only evaluated the static appearances of the obese "peer buddys" and lean "experts", and then selected one avatar for the dynamic evaluation. It could be possible that the dynamic evaluation of the obese avatars would have revealed different results than the static evaluation. Future research should address this limitation and could also evaluate the impact of the different avatars on adherence regarding the recommended daily steps or diet in a clinical trial. Second, although our results reveal preferences regarding lean "expert" avatars, using an avatar that represents a virtual doppelganger could be beneficial too and was also suggested by a patient in the free-text field. Therefore, future studies could experiment with advanced avatar technologies that are able to generate virtual doppelgangers from images of the patient. Additionally, advanced approaches could also generate images of the future self after successful weight loss transformation and could serve as a motivational tool in behavior therapy (e.g., [64]). Third, it could be possible that the obese avatars investigated in the present study were designed disadvantageous and another appearance might have resulted in more favorable ratings. Also, the lean characters but not the obese ones were wearing eye glasses, which could have contributed to the different intelligence ratings. Thus, future research could evaluate additional obese avatars with different appearances to validate the results. Given that body dissatisfactions could be one reason for the significant differences, future studies could also assess the impact of body image disorders on the avatar ratings. In addition, it seems questionable to what extent the results apply to other contexts, e.g., other ethnicities, cultures or diseases. For example, obese avatars could be well suited for a population with a BMI between 20 and 25 $\mathrm{kg} / \mathrm{m}^{2}$ [35] but inappropriate for morbidly obese patients. Subsequent research could repeat the study in other contexts. Furthermore, we limited similarity to the user by the visual appearance. However, similarity can be defined beyond and might be, for instance, also triggered with verbal cues if a lean character would say "I used to be like you...". This could be addressed in 
subsequent investigations. Finally, future studies could investigate the effects of omnipresence (e.g. perceived technostress and efficacy of the coaching) by deploying one or multiple coaches on different platforms (e.g., virtual/augmented reality glasses, mobile phone and smartwatch).

\section{References}

[1] A. Afshin et al., "Health effects of dietary risks in 195 countries, 1990-2017: a systematic analysis for the Global Burden of Disease Study 2017," The Lancet, vol. 393, no. 10184, Art. no. 10184, 2019.

[2] T. Meier, K. Senftleben, P. Deumelandt, O. Christen, K. Riedel, and M. Langer, "Healthcare Costs Associated with an Adequate Intake of Sugars, Salt and Saturated Fat in Germany: A Health Econometrical Analysis," PLOS ONE, vol. 10, no. 9, p. e0135990, Sep. 2015.

[3] G. A. Bray, "Medical Consequences of Obesity," J. Clin. Endocrinol. Metab., vol. 89, no. 6, pp. 2583-2589, Jun. 2004.

[4] WHO, Obesity: preventing and managing the global epidemic. World Health Organization, 2000.

[5] S. C. Bischoff et al., "Multicenter evaluation of an interdisciplinary 52-week weight loss program for obesity with regard to body weight, comorbidities and quality of life- a prospective study," Int. J. Obes., vol. 36, no. 4, pp. 614-624, Apr. 2012.

[6] K. Dounavi and O. Tsoumani, "Mobile Health Applications in Weight Management: A Systematic Literature Review," Am. J. Prev. Med., vol. 56, no. 6, pp. 894-903, Jun. 2019.

[7] R. A. Asbjørnsen et al., "Persuasive System Design Principles and Behavior Change Techniques to Stimulate Motivation and Adherence in Electronic Health Interventions to Support Weight Loss Maintenance: Scoping Review," J. Med. Internet Res., vol. 21, no. 6, p. e14265, Jun. 2019.

[8] H. Oinas-Kukkonen, "A foundation for the study of behavior change support systems," Pers. Ubiquitous Comput., vol. 17, no. 6, Art. no. 6, 2013.

[9] L. T. Car et al., "Conversational agents in health care: Scoping review and conceptual analysis," J. Med. Internet Res., vol. 22, no. 8, p. e17158, 2020.

[10] L. L. Kramer, S. ter Stal, B. C. Mulder, E. de Vet, and L. van Velsen, "Developing Embodied Conversational Agents for Coaching People in a Healthy Lifestyle: Scoping Review," J. Med. Internet Res., vol. 22, no. 2, p. e14058, Feb. 2020.

[11] B. A. Kamphorst, "E-coaching systems," Pers. Ubiquitous Comput., vol. 21, no. 4, Art. no. 4, 2017.

[12] D. Griol, J. Carbó, and J. M. Molina, "An automatic dialog simulation technique to develop and evaluate interactive conversational agents," Appl. Artif. Intell., vol. 27, no. 9, pp. 759-780, 2013.

[13] A.-M. Seeger, J. Pfeiffer, and A. Heinzl, "Texting with human-like conversational agents: Designing for anthropomorphism," J. Assoc. Inf. Syst. JAIS, 2020.

[14] T. Bickmore and J. Cassell, "Social dialongue with embodied conversational agents," in Advances in natural multimodal dialogue systems, Springer, 2005, pp. 23-54.

[15] K. Loveys, G. Sebaratnam, M. Sagar, and E. Broadbent, "The effect of design features on relationship quality with embodied conversational agents: a systematic review," Int. J. Soc. Robot., pp. 1-20, 2020.

[16] M. Fischer, N. Oberänder, and A. Weimann, "Four main barriers to weight loss maintenance? A quantitative analysis of difficulties experienced by obese patients after successful weight reduction," Eur. J. Clin. Nutr., vol. 74, no. 8, Art. no. 8, Aug. 2020.

[17] M. Horne et al., "Using avatars in weight management settings: A systematic review," Internet Interv., vol. 19, p. 100295, Mar. 2020.

[18] R. A. Carels, C. B. Wott, K. M. Young, A. Gumble, A. Koball, and M. W. Oehlhof, "Implicit, explicit, and internalized weight bias and psychosocial maladjustment among treatment-seeking adults," Eat. Behav., vol. 11, no. 3, pp. 180-185, Aug. 2010.

[19] P. Tropea et al., "Rehabilitation, the Great Absentee of Virtual Coaching in Medical Care: Scoping Review," J. Med. Internet Res., vol. 21, no. 10, p. e12805, Oct. 2019.

[20] C. Nass and Y. Moon, "Machines and Mindlessness: Social Responses to Computers," J. Soc. Issues, vol. 56, no. 1, Art. no. 1, Jan. 2000.

[21] H. Oinas-Kukkonen and M. Harjumaa, "Persuasive Systems Design: Key Issues, Process Model, and System Features," Commun. Assoc. Inf. Syst., vol. 24, 2009.

[22] R. M. Perloff, The dynamics of persuasion: communication and attitudes in the 21st century, 2nd ed. Mahwah, N.J: Lawrence Erlbaum Associates, 2003.

[23] D. J. O'keefe, Persuasion: Theory and research. Sage Publications, 2015.

[24] T. Kowatsch et al., "The impact of interpersonal closeness cues in text-based healthcare chatbots on attachment bond and the desire to continue interacting: an experimental design," 26th Eur. Conf. Inf. Syst. ECIS 2018, 2018.

[25] S. ter Stal, M. Tabak, H. op den Akker, T. Beinema, and H. Hermens, "Who do you prefer? The effect of age, gender and role on users' first impressions of embodied conversational agents in eHealth," Int. J. HumanComputer Interact., vol. 36, no. 9, Art. no. 9, 2020.

[26] T. W. Liew, S.-M. Tan, and C. Jayothisa, "The effects of peer-like and expert-like pedagogical agents on learners' agent perceptions, task-related attitudes, and learning achievement," J. Educ. Technol. Soc., vol. 16, no. 4, Art. no. 4, 2013.

[27] R. B. Rosenberg-Kima, A. L. Baylor, E. A. Plant, and C. E. Doerr, "Interface agents as social models for female students: The effects of agent visual presence and appearance on female students' attitudes and beliefs," Comput. Hum. Behav., vol. 24, no. 6, Art. no. 6, Sep. 2008.

[28] A. Bandura, Social foundations of thought and action: a social cognitive theory. Prentice-Hall, 1986.

[29] D. E. Byrne, The attraction paradigm, vol. 462. Academic press, 1971.

[30] A. Bandura, Self-efficacy: the exercise of control. New 
York: W.H. Freeman, 1997.

[31] Y. Kim and A. L. Baylor, "Research-Based Design of Pedagogical Agent Roles: a Review, Progress, and Recommendations," Int. J. Artif. Intell. Educ., vol. 26, no. 1 , Art. no. 1, 2016.

[32] R. B. Kantharaju, D. De Franco, A. Pease, and C. Pelachaud, "Is Two Better than One?: Effects of Multiple Agents on User Persuasion," in Proceedings of the 18th International Conference on Intelligent Virtual Agents, Sydney NSW Australia, Nov. 2018, pp. 255-262.

[33] J. Fox and J. N. Bailenson, "Virtual self-modeling: The effects of vicarious reinforcement and identification on exercise behaviors," Media Psychol., vol. 12, no. 1, Art. no. 1, 2009.

[34] J. Navarro, A. Cebolla, R. Llorens, A. Borrego, and R. M. Baños, "Manipulating Self-Avatar Body Dimensions in Virtual Worlds to Complement an Internet-Delivered Intervention to Increase Physical Activity in Overweight Women," Int. J. Environ. Res. Public. Health, vol. 17, no. 11, Art. no. 11, Jun. 2020.

[35] H. C. van Vugt, E. A. Konijn, J. F. Hoorn, and J. Veldhuis, "Why fat interface characters are better ehealth advisors," in International Workshop on Intelligent Virtual Agents, 2006, pp. 1-13.

[36] M. A. Napolitano, S. Hayes, G. Russo, D. Muresu, A. Giordano, and G. D. Foster, "Using avatars to model weight loss behaviors: participant attitudes and technology development," J. Diabetes Sci. Technol., vol. 7, no. 4, Art. no. 4, 2013.

[37] A. Gulz and M. Haake, "Design of animated pedagogical agents-A look at their look," Int. J. Hum.-Comput. Stud., vol. 64, no. 4, pp. 322-339, Apr. 2006.

[38] S. ter Stal, L. L. Kramer, M. Tabak, H. op den Akker, and H. Hermens, "Design Features of Embodied Conversational Agents in eHealth: a Literature Review," Int. J. Hum.-Comput. Stud., vol. 138, p. 102409, Jun. 2020.

[39] M. Mori, K. MacDorman, and N. Kageki, "The Uncanny Valley [From the Field]," IEEE Robot. Autom. Mag., vol. 19, no. 2, Art. no. 2, Jun. 2012.

[40] C.-C. Ho and K. F. MacDorman, "Revisiting the uncanny valley theory: Developing and validating an alternative to the Godspeed indices," Comput. Hum. Behav., vol. 26, no. 6, Art. no. 6, 2010.

[41] A. R. Hevner, "A three cycle view of design science research," Scand. J. Inf. Syst., vol. 19, no. 2, Art. no. 2, 2007.

[42] S. Gregor and D. Jones, "The Anatomy of a Design Theory," J. Assoc. Inf. Syst., vol. 8, no. 5, pp. 312-335, May 2007.

[43] T. Weimann, H. Schlieter, and M. Fischer, "Designing an Avatar-Based Virtual Coach for Obesity Patients," in The Next Wave of Sociotechnical Design, vol. 12807, L. Chandra Kruse, S. Seidel, and G. I. Hausvik, Eds. Cham: Springer International Publishing, 2021, pp. 5257.

[44] A. Cafaro, H. H. Vilhjálmsson, and T. Bickmore, "First Impressions in Human-Agent Virtual Encounters," ACM Trans. Comput.-Hum. Interact. TOCHI, vol. 23, no. 4, Art. no. 4, 2016.
[45] K. Bergmann, F. Eyssel, and S. Kopp, "A second chance to make a first impression? How appearance and nonverbal behavior affect perceived warmth and competence of virtual agents over time," in International conference on intelligent virtual agents, 2012, pp. 126-138.

[46] C. Bartneck, D. Kulić, E. Croft, and S. Zoghbi, "Measurement instruments for the anthropomorphism, animacy, likeability, perceived intelligence, and perceived safety of robots," Int. J. Soc. Robot., vol. 1, no. 1, Art. no. 1, 2009.

[47] S. ter Stal, M. Broekhuis, L. van Velsen, H. Hermens, and M. Tabak, "Embodied Conversational Agent Appearance for Health Assessment of Older Adults: Explorative Study," JMIR Hum. Factors, vol. 7, no. 3, Art. no. 3, Sep. 2020.

[48] H. op den Akker et al., "Council of Coaches - A Novel Holistic Behavior Change Coaching Approach," in Proceedings of the 4th International Conference on Information and Communication Technologies for Ageing Well and e-Health - Volume 1: ICT4AWE, 2018, pp. 219-226.

[49] A. J. Cowell and K. M. Stanney, "Embodiment and Interaction Guidelines for Designing Credible, Trustworthy Embodied Conversational Agents," in Intelligent Virtual Agents, vol. 2792, T. Rist, R. S. Aylett, D. Ballin, and J. Rickel, Eds. Berlin, Heidelberg: Springer Berlin Heidelberg, 2003, pp. 301-309.

[50] A. L. Baylor, "The design of motivational agents and avatars," Educ. Technol. Res. Dev., vol. 59, no. 2, Art. no. 2, Apr. 2011.

[51] D. Parmar, S. Olafsson, D. Utami, and T. Bickmore, "Looking the Part: The Effect of Attire and Setting on Perceptions of a Virtual Health Counselor," in Proceedings of the 18th International Conference on Intelligent Virtual Agents, Sydney NSW Australia, Nov. 2018, pp. 301-306.

[52] S. Gregor, L. Chandra Kruse, and S. Seidel, "Research Perspectives: The Anatomy of a Design Principle," $J$. Assoc. Inf. Syst., vol. 21, no. 6, Art. no. 6, 2020.

[53] V. Schwind, K. Wolf, and N. Henze, "Avoiding the uncanny valley in virtual character design," Interactions, vol. 25, no. 5, Art. no. 5, Aug. 2018.

[54] E. Hudlicka, "Virtual training and coaching of health behavior: Example from mindfulness meditation training," Patient Educ. Couns., vol. 92, no. 2, Art. no. 2, Aug. 2013.

[55] K. F. MacDorman, R. D. Green, C.-C. Ho, and C. T. Koch, "Too real for comfort? Uncanny responses to computer generated faces," Comput. Hum. Behav., vol. 25, no. 3, Art. no. 3, 2009.

[56] A. L. Baylor, "Promoting motivation with virtual agents and avatars: role of visual presence and appearance," Philos. Trans. R. Soc. B Biol. Sci., vol. 364, no. 1535, Art. no. 1535, Dec. 2009.

[57] V. Gamage and C. Ennis, "Examining the effects of a virtual character on learning and engagement in serious games," in Proceedings of the 11th Annual International Conference on Motion, Interaction, and Games, Limassol Cyprus, Nov. 2018, pp. 1-9. 
[58] J. Xiao, J. Stasko, and R. Catrambone, "The role of choice and customization on users' interaction with embodied conversational agents: effects on perception and performance," in Proceedings of the SIGCHI conference on Human factors in computing systems, 2007, pp. 1293-1302.

[59] P. Virtanen et al., "SciPy 1.0: Fundamental Algorithms for Scientific Computing in Python," Nat. Methods, vol. 17, pp. 261-272, 2020.

[60] P. Kulms, N. C. Krämer, J. Gratch, and S.-H. Kang, "It's in their eyes: A study on female and male virtual humans' gaze," in International workshop on intelligent virtual agents, 2011, pp. 80-92.

[61] J. Feine, U. Gnewuch, S. Morana, and A. Maedche, "Gender Bias in Chatbot Design," in Chatbot Research and Design, vol. 11970, A. Følstad, T. Araujo, S. Papadopoulos, E. L.-C. Law, O.-C. Granmo, E. Luger, and P. B. Brandtzaeg, Eds. Cham: Springer International Publishing, 2020, pp. 79-93.

[62] C. Sikorski et al., "The stigma of obesity in the general public and its implications for public health-a systematic review," BMC Public Health, vol. 11, no. 1, Art. no. 1, 2011.

[63] N.-A. Weinberger, A. Kersting, S. G. Riedel-Heller, and C. Luck-Sikorski, "Body Dissatisfaction in Individuals with Obesity Compared to Normal-Weight Individuals: A Systematic Review and Meta-Analysis," Obes. Facts, vol. 9, no. 6, Art. no. 6, 2016.

[64] G. Ossolinski, M. Jiwa, A. McManus, and R. Parsons, "Do images of a personalised future body shape help with weight loss? A randomised controlled study," Trials, vol. 18, no. 1, Art. no. 1, 2017. 